\title{
Anti-tumor Activity of the Novel Hexahydrocannabinol Analog LYR-8 in Human Colorectal Tumor Xenograft Is Mediated through the Inhibition of Akt and Hypoxia-Inducible Factor-1 $\alpha$ Activation
}

\author{
Dinesh Thapa $,{ }^{a},{ }^{\dagger}$ Youra Kang, ${ }^{a}$ Pil-Hoon Park, ${ }^{a}$ Seok Kyun Noh, ${ }^{b}$ Yong Rok Lee, ${ }^{*, b}$ \\ Sung Soo Han, ${ }^{c}$ Sae Kwang Ku, ${ }^{d}$ Yunjin Jung, ${ }^{e}$ and Jung-Ae Kim*,a \\ ${ }^{a}$ College of Pharmacy, Yeungnam University; ${ }^{b}$ School of Chemical Engineering and Technology, Yeungnam Univer- \\ sity; ${ }^{c}$ Department of Nano, Medical \& Polymer Materials, College of Engineering, Yeungnam University; Gyeongsan \\ 712-749, Republic of Korea: ${ }^{d}$ College of Oriental Medicine, Daegu Hanny University; Gyeongsan 712-715, Republic \\ of Korea: and ${ }^{e}$ College of Pharmacy, Pusan National University; Busan 609-735, Republic of Korea. \\ Received January 2, 2012; accepted March 21, 2012
}

Cannabinoid compounds have been shown to exert anti-tumor effects by affecting angiogenesis, invasion, and metastasis. In the present study, we examined the action mechanism by which LYR-8, a novel hexahydrocannabinol analog, exerts anti-angiogenic and anti-tumor activity in human cancer xenografts. In the xenografted tumor tissues, LYR-8 significantly reduced the expression of hypoxia-inducible factor-1 alpha (HIF-1 $\alpha$ ), a transcription factor responsible for induction of angiogenesis-promoting factors, and its target genes, vascular endothelial growth factor (VEGF) and cyclooxygenase-2 (COX-2). In HT-29 human colon cancer cells treated with a hypoxia-inducing agent $\left(\mathrm{CoCl}_{2}\right)$, LYR-8 dose-dependently suppressed the induction of HIF- $1 \alpha$ and subsequently its targets, VEGF and COX-2. In addition, highly elevated prostaglandin $\mathbf{E}_{2}$ $\left(\mathrm{PGE}_{2}\right)$ concentrations in $\mathrm{CoCl}_{2}$-treated HT-29 cells were also significantly suppressed by LYR-8. However, LYR-8 alone in the absence of $\mathrm{CoCl}_{2}$ did not alter the basal expression of VEGF and COX-2, or PGE pro- $_{2}$ duction. Furthermore, LYR-8 effectively suppressed Akt signaling, which corresponded to the suppression of $\mathrm{CoCl}_{2}$-induced HIF-1 $\alpha$ accumulation. Taken together, LYR-8 exerts anti-tumor effects through the inhibition of Akt and HIF-1 $\alpha$ activation, and subsequently suppressing factors regulating tumor microenvironment, such as VEGF and COX-2. These results indicate a novel function of cannabinoid-like compound LYR-8 as an anti-tumor agent with a HIF-1 $\alpha$ inhibitory activity.

Key words anti-tumor; hexahydrocannabinol; Akt; hypoxia-inducible factor- $1 \alpha$; vascular endothelial growth factor; cyclooxygenase-2

Angiogenesis, the formation of new blood vessels, is a critical factor not only in physiological processes but also in numerous diseases including cancer. ${ }^{1,2)}$ Increasing insight and understanding of the biology of the excessive and abnormal blood vessels in tumors has led to a focus on angiogenesis signaling molecules as cancer therapeutic targets. Vascular endothelial growth factor (VEGF) is produced in response to hypoxic stimuli triggered by conditions like tumor growth. Indeed, the role of VEGF in tumor angiogenesis has been translated into the clinic, as VEGF inhibitors such as bevacizumab, have been used for the treatment of solid tumors. ${ }^{3)}$ However, because tumors have ability to secrete a variety of pro-angiogenic factors, the clinical efficacy of anti-VEGF anti-angiogenic agents is transient, and tumors eventually resume aggressive growth. Recent studies on anti-angiogenic anti-tumor drug development have focused on the molecules governing upstream proangiogenic factor production.

In tumor microenvironments, VEGF is mainly regulated by hypoxia-inducible factors (HIFs), which are heterodimeric transcription factors. HIF-1 is one of the best studied members of this family and is composed of HIF- $1 \alpha$ and aryl hydrocarbon receptor nuclear translocator (ARNT). ${ }^{4,5)}$ The HIF-1 subunits are constitutively expressed at the transcriptional and translational levels, but the HIF- $1 \alpha$ component is regulated in an $\mathrm{O}_{2}$-dependent manner via ubiquitin-proteosomal

The authors declare no conflict of interest.

${ }^{\dagger}$ Present address: Department of Urology, School of Medicine, University of Texas Health Science Center at San Antonio; San Antonio, TX, U.S.A. degradation. ${ }^{6)}$ HIF- $1 \alpha$ is upregulated in a wide variety of human primary tumors compared to corresponding normal tissues. $^{7-9)}$ Furthermore, the binding of HIF-1 $\alpha$ to the hypoxia response element (HRE) leads to the transcriptional regulation of several genes involved in tumor angiogenesis including VEGF. ${ }^{10)}$ In addition to its role in angiogenesis, HIF- $1 \alpha$ also regulates a large number of genes that are extensively involved in tumor survival, aggressive progression and drug resistance. ${ }^{11)}$ Therefore, HIF-1 $\alpha$ and other factors interacting with HIF-1 $\alpha$ have been suggested as therapeutic targets for anti-cancer drug development. ${ }^{12,13)}$

We recently synthesized and evaluated the anti-cancer effects of a novel series of hexahydrocannabinol analogs. ${ }^{14-16)}$ Of these analogs, LYR-8, a cannabinoid-like compound with no affinity for conventional cannabinoid receptors $\left(\mathrm{CB}_{1}\right.$ and $\left.\mathrm{CB}_{2}\right)$, was found to directly inhibit the growth, induce apoptosis of cancer cells and inhibit endothelial cell proliferation and angiogenesis. In addition, we observed that the apoptotic activity of LYR-8 in colon cancer cells was mediated by p53-independent NAG-1 activation and by the induction of other apoptotic markers such as p21 and caspase-3. ${ }^{15}$ LYR-8 was also found to have anti-proliferative effects on endothelial cells and other cancer cell lines, including drug-resistant breast cancer cells. Furthermore, in subsequent in vitro and in vivo assays, we found that LYR-8 significantly and dose-dependently inhibited VEGF-induced endothelial cell migration, invasion, tube formation, and neovascularization. ${ }^{16)}$ However, in vivo anticancer efficacy and the molecular signaling pathway of the activity of LYR-8 have not been elucidated. 
In the present study, we explored the mechanism by which LYR-8 exerts its anti-angiogenic anti-tumor effects in in vitro HT-29 cells treated with $\mathrm{CoCl}_{2}$ (a hypoxia mimetic agent) and in vivo models using xenograft tumor in mice and chick chorioallantoic membrane (CAM) implanted with cancer cells (HT-29).

\section{MATERIALS AND METHODS}

Chemical Synthesis and Treatment of LYR-8 LYR-8 was synthesized as described previously. ${ }^{14)}$ Its chemical structure is shown in Fig. 1. A $50 \mathrm{~mm}$ stock solution of LYR-8 was prepared in dimethyl sulfoxide (DMSO), stored at $-20^{\circ} \mathrm{C}$, and then diluted as needed. For in vitro incubations, LYR- 8 was directly applied at a final DMSO concentration of $0.1-0.2 \%$ (v/v). For in vivo experiments (CAM tumor and tumor xenograft implantation in mice), LYR-8 was prepared in ethanol and diluted with phosphate buffered saline (PBS) supplemented with $0.1 \%$ bovine serum albumin (BSA). The vehicles had no significant effect on any of the parameters assessed.

Cell Culture HT-29 (human colon cancer) cell line was obtained from American Type Culture Collection (Manassas, VA, U.S.A.). The cells were grown in standard growth medium supplemented with $10 \%$ fetal bovine serum (FBS) and $1 \%$ penicillin/streptomycin, and maintained at $37^{\circ} \mathrm{C}$ in a $5 \% \mathrm{CO}_{2}$-humidified atmosphere. Culture media were replaced every $2 \mathrm{~d}$.

Mouse Model of Tumor Xenograft Growth and Angiogenesis All experiments on mice were performed according to the guidelines issued by the Yeungnam University Research Committee for the Care and Use of Laboratory Animals. Mice were maintained under pathogen-free conditions and a 12-h light/12-h dark cycle. HT-29 cells $\left(5 \times 10^{6}\right)$ suspended in serum-free medium were injected subcutaneously into the rear flanks of six week-old BALB/c nude mice. After tumors had grown to $c a .50 \mathrm{~mm}^{3}$, mice were randomly assigned to two groups and intraperitoneally treated with LYR-8 (10 mg/ $\mathrm{kg} / \mathrm{d}$ ) or vehicle. The body weights and clinical observations were recorded daily. The tumors were measured with a digital caliper, and the volumes calculated using the formula $V=(\text { width })^{2} \times$ length $/ 2$. Mice were euthanized $18 \mathrm{~d}$ after drug treatment using diethyl ether and solid tumors were removed and processed for further analyses.

Histomorphometry and Quantification of Immunostaining For histological examination, tumor samples were fixed in $10 \%$ neutral buffered formalin. After paraffin embedding, tissue sections of $4 \mu \mathrm{m}$ thickness were prepared and stained with hematoxylin and eosin (H\&E) for examination by light microscopy. After that, the histological profiles of individual cross-trimmed tumor masses were determined. Masson's trichrome staining was performed for the detection of tumor cells with collagen fiber. Azocarmine G (AZAN) staining was performed to visualize the basement membranes and erythrocytes, representing the presence of blood vessels. The regions occupied by tumor cells percentages of tumor cross sections) and the numbers of vessels (number $/ \mathrm{mm}^{2}$ at mass peripheries) were quantified on the prepared individual histological tumor samples using a digital image analyzer (DMI300, DMI, Korea). For immunohistochemistry, tissue sections were subjected to citrate buffer antigen (epitope) retrieval ${ }^{17}$ ) and incubated with methanol and $0.3 \% \mathrm{H}_{2} \mathrm{O}_{2}$ for $30 \mathrm{~min}$ to

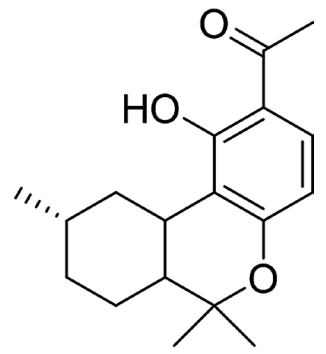

\section{LYR-8}

Fig. 1. The Chemical Structure of LYR-8

inhibit endogenous peroxidase activity at room temperature. After blocking with horse serum (Vector Laboratories Inc., CA, U.S.A.; dilution $1: 100$ ), the sections were incubated with rabbit polyclonal primary antibodies against HIF- $1 \alpha$ (dilution $1: 200$ ), VEGF (dilution $1: 100$ ) or COX-2 (dilution $1: 200$ ) at $4{ }^{\circ} \mathrm{C}$ overnight in a humidity chamber. The sections were then incubated with biotinylated universal secondary antibody (Vector Lab. Inc.; dilution 1:50) followed by avidin-biotin complex reagents (Vectastain Elite ABC Kit, Vector Lab. Inc.; dilution 1:50) and counterstained with Mayer's hematoxylin solution. The numbers of positive cells per 100 tumor cells were calculated using an automatic image analyzer. The histopathologist was unaware of the sample identities when the analyses were performed. Histomorphometrical analysis was performed for 3 fields per tumor mass (total of 9 fields/group) at a magnification of $\times 400$.

The in Vivo Human Cancer Xenograft Model Using Chick Chorioallantoic Membrane (CAM) CAM preparation was carried out as described previously. ${ }^{18)}$ For tumor implantation, cancer cells grown in monolayers were trypsinized, washed, and re-suspended in serum-free RPMI 1640 medium at $4{ }^{\circ} \mathrm{C}$. The cells were then mixed with $50 \%(\mathrm{v} / \mathrm{v}) \mathrm{GFR}-$ Matrigel in the absence or presence of LYR-8. The mixed cell suspension $(30 \mu \mathrm{L})$ was then loaded directly onto CAMs. After incubation for $5 \mathrm{~d}, \mathrm{CAM}$ tissues around areas of implantation were resected from the embryos and photographed under a light microscope (Leica, Germany). The tumors were then carefully removed without other adjacent CAM tissue, weighed, snap-frozen in liquid nitrogen, and stored at $-80^{\circ} \mathrm{C}$. The regions occupied by tumor cells (percentage over crossly trimmed tumor mass) and the numbers of vessels (number/ $\mathrm{mm}^{2}$ of marginal regions of tumor masses) were quantified on the prepared individual histological tumor samples using a digital image analyzer (DMI-300, DMI, Korea).

Protein Extraction and Western Blotting The whole cell lysates were prepared using RIPA buffer, and the protein contents were measured with the bicinchoninic acid (BCA) protein assay reagent (Pierce, Rockford, IL, U.S.A.). Equal amounts of total proteins were separated by sodium dodecyl sulfate-polyacrylamide gel electrophoresis (SDS-PAGE) and transferred onto Hybond ECL nitrocellulose membranes (Amersham Life Science, Buckinghamshire, U.K.) at $200 \mathrm{~mA}$ for $1 \mathrm{~h}$. The membranes were blocked with 5\% skim milk in Trisbuffered saline (TBS)-Tween 20 (TBS-T) at room temperature for $1 \mathrm{~h}$, and then incubated with specific antibodies in skim milk-TBS at $4^{\circ} \mathrm{C}$ overnight. The membranes were then washed three times with TBS-T and incubated with horseradish 
A

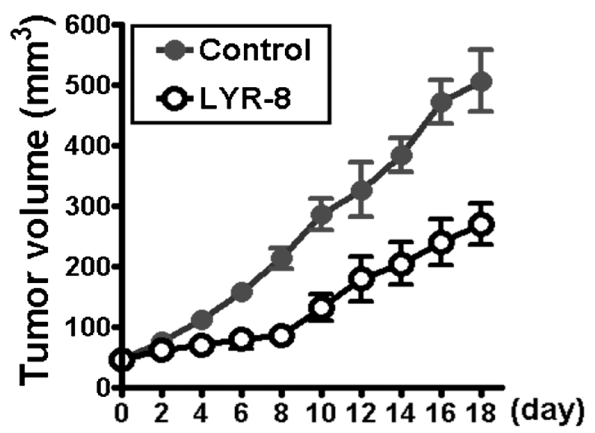

C

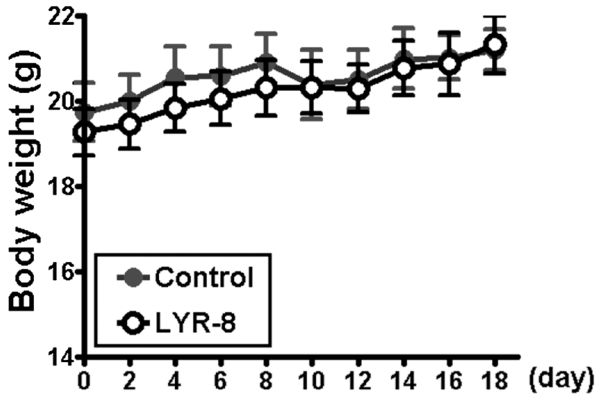

B
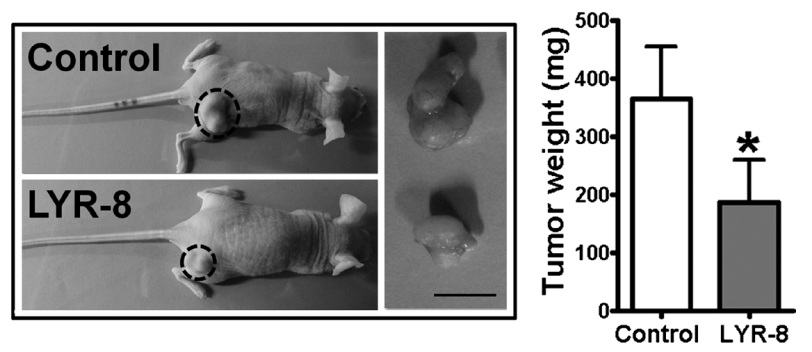

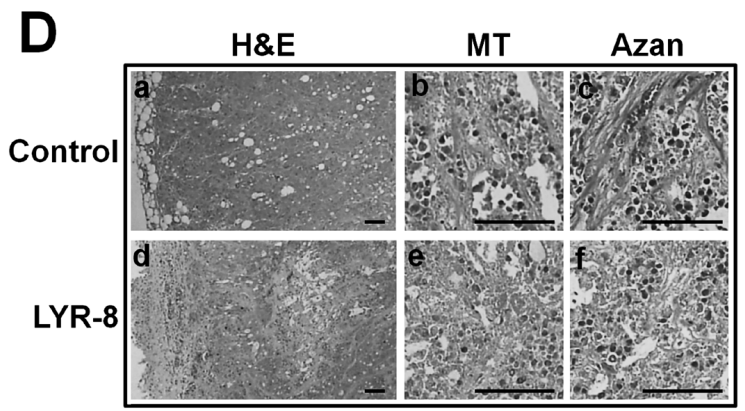

$E$

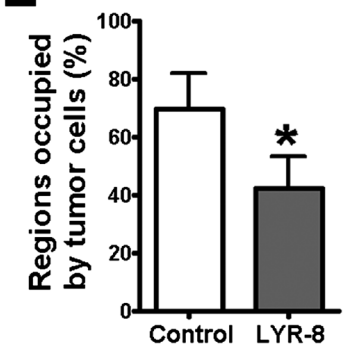

F

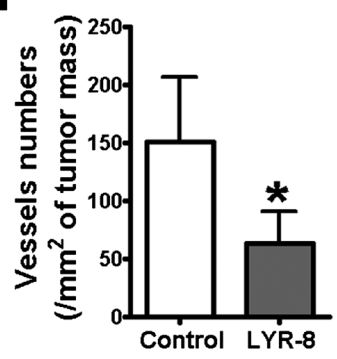

G

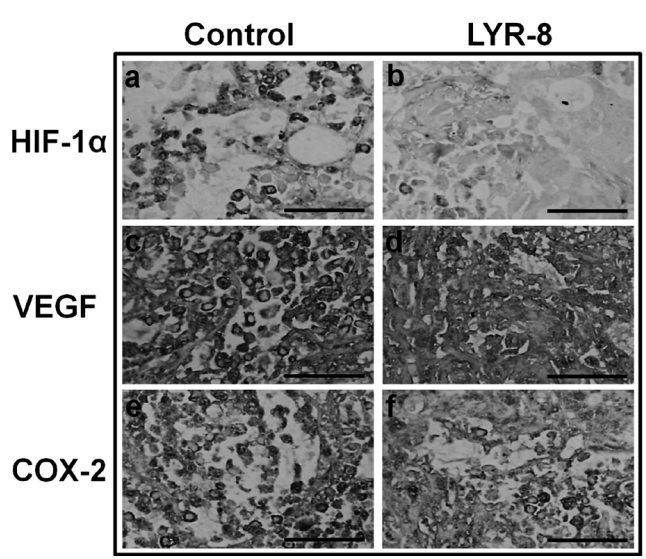

$\mathrm{H}$

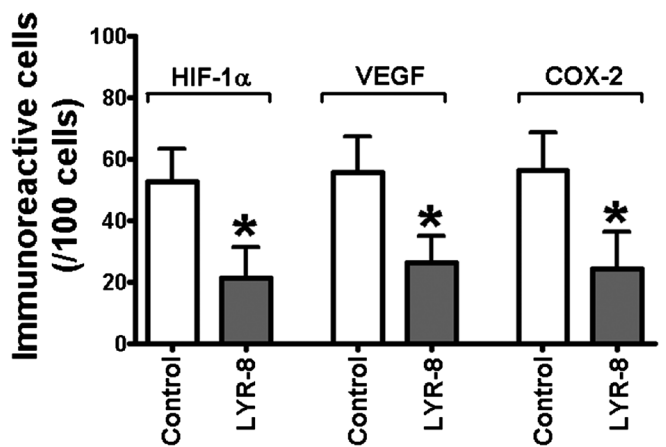

Fig. 2. LYR-8 Suppresses Tumor Growth, Angiogenesis, and the Expression Level of HIF-1 $\alpha$, VEGF and COX-2 in a Mouse Xenograft Model

(A) HT-29 cells were injected subcutaneously into the right flank of 6-week-old male athymic nude mice, and tumors were allowed to grow to $50 \mathrm{~mm}{ }^{3}$. LYR-8 $(10 \mathrm{mg} /$ $\mathrm{kg}$ ) or PBS was then administered via intraperitoneal injection. ( $n=6$ animals/group) daily from day 0 to day 17 . Tumor volumes were calculated using the longest diameter $\times$ width ${ }^{2} \times 0.5$. (B) On day 18 , mice treated with PBS or LYR-8 were euthanized (left) and tumors were excised (right). The tumors were imaged using a digital camera. The bar graph represents the average tumor weight \pm S.E.M. (C) The changes in body weight were measured from day 0 to day 17 during treatment with vehicle (ethanol in PBS) or LYR-8 $(n=6)$. (D-H) The xenograft tumor tissue was removed from mice, embedded in paraffin, and analyzed by light microscopic analysis and immunohistochemistry: After staining using three different protocols [H\&E, Masson's trichrome (MT), or AZAN] descriptive light microscopic analysis was performed to evaluate the morphologic changes in tumor cell densities and collagen matrices (D). Each panel represents H\&E staining (a,d), regions occupied by MT-stained tumor cells $(\mathrm{b}, \mathrm{e})$, and blood vessels stained with AZAN (c,f). Quantitative data for tumor cells in tumor sections (E) and number of blood vessels per $\mathrm{mm}^{2}$ of the central region of the tumor sections (F) were obtained using a digital image analyzer. $* p<0.05$, compared to the vehicle-treated control group. Scale bars $=160 \mu \mathrm{m}$. (G-H), Immunohistochemical analysis. Tumor sections were stained (G) with antibodies against HIF-1 $\alpha$ (a,b), VEGF (c,d) or COX-2 (e,f). Numbers of immune-positive cells among 100 tumor cells in the center region of the tumor mass were counted $(\mathrm{H}) .{ }^{*} p<0.05$, compared to the vehicle-treated control group. Scale bars $=320 \mu \mathrm{m}$. 
peroxidase-conjugated secondary antibody in skim milk-TBS for $1 \mathrm{~h}$ at room temperature. The immunoreactive proteins were visualized using an ECL kit (Pierce) and digitally processed using a LAS-4000 mini unit (Fuji, Japan). The membranes were stripped and reprobed with an actin antibody as a loading control. Densitometric analyses of the blots were performed using Multi Gauge Ver 3.2 imaging software in a Fuji Image Station.

RNA Isolation and Quantitative Real Time Polymerase Chain Reaction (qRT-PCR) The cells were collected and total RNA was extracted using RNeasy kits (Qiagen, Hilden, Germany). The isolated mRNAs were reverse transcribed and gene expression was analyzed by qRT-PCR using a RotorGene 6000 (Corbett, Sydney, Australia). The primer sequences for VEGF were as follows: sense 5'-CCTGGTGGACATCTT CCAGGAGTACC-3' and antisense 5'-GAAGCTCATCTCTCC TATGTGCTGGC-3'. The reaction mixture consisted of $2 \mu \mathrm{L}$ of cDNA template, $10 \mu \mathrm{L}$ of SYBR Green PCR master mix, and $5 \mathrm{pm}$ of primers in a total volume of $20 \mu \mathrm{L}$. The cDNA was denatured at $95^{\circ} \mathrm{C}$ for $15 \mathrm{~min}$ and amplified over 40 cycles $\left(95^{\circ} \mathrm{C}\right.$ for $5 \mathrm{~s}, 55^{\circ} \mathrm{C}$ for $10 \mathrm{~s}$ and $72^{\circ} \mathrm{C}$ for $\left.20 \mathrm{~s}\right)$. GAPDH was used as an internal control. The fold changes over control levels were determined using the comparative cycle threshold method.

VEGF and Prostaglandin $\mathrm{E}_{2} \quad\left(\mathrm{PGE}_{2}\right)$ Immunoassays Secreted VEGF levels were quantified using a Quantikine human VEGF ELISA kit (R\&D Systems, Minneapolis, MN, U.S.A.). $\mathrm{PGE}_{2}$ concentrations in cell culture media were determined using a commercially available enzyme immunoassay kit (Cayman Chemical, Ann Arbor, MI, U.S.A.). In brief, HT29 cells were seeded in 24-well plates and grown to $80-90 \%$ confluence. The media were them replaced with fresh serumfree medium containing $200 \mu \mathrm{M} \mathrm{CoCl}$ in the absence or presence of LYR- 8 and incubated for $18 \mathrm{~h}$. The supernatants were collected and the cells were subjected to 3-(4,5-dimethylthiazol-2-yl)-2,5-diphenyltetrazolium bromide (MTT) assays. The concentrations of VEGF and $\mathrm{PGE}_{2}$ in unknown samples were determined by comparing the optical densities of samples to a standard curve and normalized with respect to cell viability.

Statistical Analysis The data are presented as the means \pm S.E. The statistical analyses were performed with a Student's $t$ test or one-way analysis of variance (ANOVA) followed by the Student-Newman-Keuls test (GraphPad Prism4.0 software, San Diego, CA, U.S.A.) to calculate the differences between groups. $p$ values of $<0.05$ were considered statistically significant.

\section{RESULTS}

LYR-8 Administration Inhibits Tumor Growth and Angiogenesis in a Xenograft Mouse Model We investigated the antitumor potential of LYR-8 in an in vivo HT-29 tumor xenograft model. At $10 \mathrm{mg} / \mathrm{kg}$, LYR- 8 induced significant inhibition of tumor growth compared with vehicle-treated controls (10\% ethanol in PBS) (Fig. 2A). On day 18 after the first drug treatment, tumor growth was approximately 2-fold lower in the LYR-8-treated group (mean relative tumor volume $\left.271 \pm 33 \mathrm{~mm}^{3}\right)$ than in the control group $\left(507 \pm 51 \mathrm{~mm}^{3}\right)$. Gross observations, dissected tumor masses, and tumor weights confirmed that LYR-8 significantly inhibited tumor growth (Fig. 2B). Furthermore, tumors from mice injected with LYR-8 were obviously paler than tumors from vehicle control-treated groups, which we attribute to a reduction in blood vessel intensity (Fig. 2B). Importantly, and in-line with our previous observation that LYR-8 does not bind to either the $\mathrm{CB}_{1}$ or $\mathrm{CB}_{2}$ receptors, ${ }^{15)}$ LYR-8 did not induce any psychoactive behavior in the mice. In addition, it had no significant effect on body weight (Fig. 2C), indicating a lack of toxicity under the test conditions. Histologic and morphometric analysis of the tumor sections by Masson's trichrome staining revealed that LYR-8 suppressed tumor cell density (Fig. 2D MT panel and 2E). Compared with vehicle-treated controls, the number of blood vessels that were counted in an erythrocyte-visualizing AZAN staining was also decreased by LYR- 8 treatment (Fig. 2D AZAN panel and 2F). Furthermore, immunohistochemical staining showed that the HIF-1 $\alpha$, VEGF and cyclooxygenase-2 (COX-2) levels were downregulated in the tumors of mice treated with LYR-8 versus vehicle controls (Figs. 2G,H), indicating that downregulation of HIF-1 $\alpha$ corresponds to the reduction of tumor growth and angiogenesis by LYR-8.

LYR-8 Inhibits COX-2 and VEGF Expressions in Tumors Grown on Top of CAM We implanted HT-29 cancer cells onto the primitive capillary network of the CAM to confirm that suppressed tumor growth by LYR-8 corresponds to the reduced expression of proangiogenic factors in the implanted tumor tissues. As shown in Fig. 3A, the implantation of cancer cells in CAMs dramatically induced angiogenesis, as shown by the presence of a large number of vessels growing around the area of implantation. However, after exposure to LYR-8 (144 ng/CAM once at the time of implantation), new blood vessel formation was markedly reduced (Fig. 3A). Along with suppressed angiogenesis, the weights of excised-tumors were significantly reduced by LYR-8 (Fig. 3A). Observations of chick embryo viability and resected CAM morphologies confirmed that LYR-8 was non-toxic at the test dose (data not shown).

We next performed H\&E staining and morphometric analysis on sections of HT-29 tumors grown on CAM. The results obtained showed a decrease in tumor cell density in the tumor treated with LYR-8 (Fig. 3B). HT-29 tumors obtained from CAM implants were further investigated to identify the factors responsible for inhibiting tumor growth and angiogenesis in LYR-8 treated tumors. Extracts of tumor tissues were further analyzed for VEGF and COX-2 expression, as increased levels of these genes have been linked to induction of tumor angiogenesis. LYR-8 treatment significantly downregulated VEGF and COX-2 levels but not COX-1 (Fig. 3C).

LYR-8 Inhibits Cobalt Chloride-Induced HIF-1 $\alpha$ Activation and Target Gene Expression in HT-29 Colon Cancer Cells Because cancer cells release angiogenic growth factors including VEGF in a HIF-1 $\alpha$-dependent manner, we examined whether LYR-8 exerts an inhibitory effect on induction of HIF- $1 \alpha$ and its targets VEGF and COX-2. First, we used $\mathrm{CoCl}_{2}$ to induce HIF-1 $\alpha$ overexpression based upon our previous finding that HIF-1 $\alpha$ level increases in HT-29 cells treated with $\mathrm{CoCl}_{2}$ in the same manner as cells cultured in a $1 \% \mathrm{O}_{2}$ atmosphere. ${ }^{19)}$ As shown in Fig. $4 \mathrm{~A}, \mathrm{CoCl}_{2}$ was found to dosedependently increase the level of HIF- $1 \alpha$ and VEGF in HT-29 cells cultured in the presence of different amounts of $\mathrm{CoCl}_{2}$ in serum free medium. The increase in VEGF expression level by $200 \mu \mathrm{M}$ of $\mathrm{CoCl}_{2}$ was approximately 4 -fold, corresponding to the increase in HIF-1 $\alpha$ level (Fig. 4B). Treatment of HT-29 

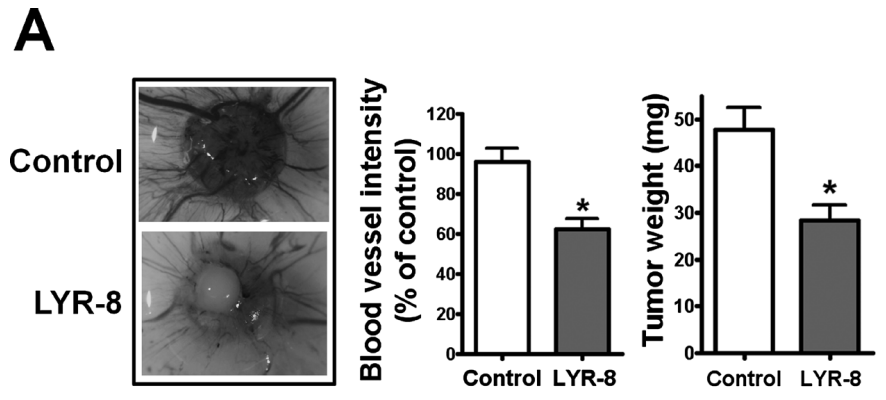

B
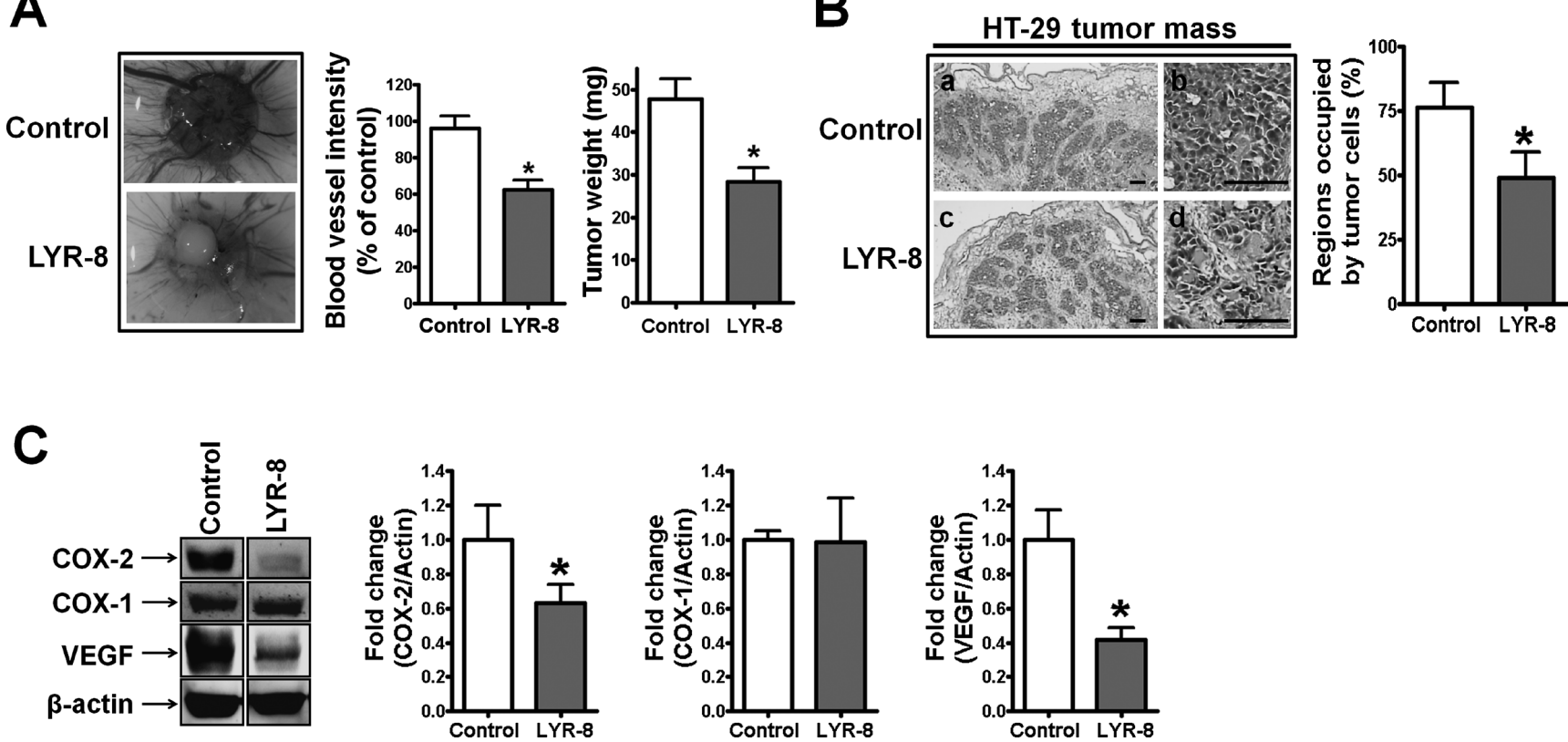

Fig. 3. LYR-8 Suppresses Markers of Angiogenesis in HT-29 CAM-Implanted Tumors

(A) The HT-29 cancer cells were loaded $\left(1.5 \times 10^{6}\right.$ cells/CAM) onto CAMs and a single dose (144ng) of LYR- 8 was administered at the time of implantation. Five days after implantation, CAMs were resected and imaged under the microscope. Tumor vasculature, quantitation of vessel numbers and tumor weights were analyzed. (B) The harvested HT-29 CAM tumor masses were fixed, embedded in paraffin and stained with H\&E. The representative histopathological profiles of tumor masses from control $(a, b)$ and LYR-8-treated samples (c,d). The tumor sections treated with LYR-8 showed significantly lower tumor cell densities than controls (right panel, bar graph). Scale bars $=80 \mu \mathrm{m}$. (C) Total cell lysates were extracted from tumors per group and analyzed for COX-1, COX-2 and VEGF expression by Western blot. The bar graphs on the right panel show the relative band density normalized to actin. ${ }^{*} p<0.05$, compared to vehicle-treated control group. $* p<0.05$, compared to vehicle-treated cancer cellimplanted group.

cells with $10 \mu \mathrm{M}$ LYR-8 in the presence of $\mathrm{CoCl}_{2}$ completely inhibited HIF- $1 \alpha$ protein accumulation without any alteration in the mRNA level (Fig. 4C). To examine the effect of LYR-8 on the stability of HIF-1 $\alpha$, cycloheximide was introduced to the $\mathrm{CoCl}_{2}$-treated cells. As shown in Fig. 4D, after protein synthesis was blocked by cycloheximide, the $\mathrm{CoCl}_{2}$-induced HIF-1 $\alpha$ level was not altered by LYR- 8 . However, $\mathrm{CoCl}_{2}-$ induced VEGF mRNA expression (Fig. 4E) and protein secretion (Fig. 4F) were strongly decreased by LYR-8 in HT-29 cells. Furthermore, highly elevated COX-2 expression (Fig. $4 \mathrm{G}$ ) and $\mathrm{PGE}_{2}$ concentration (Fig. $4 \mathrm{H}$ ) in $\mathrm{CoCl}_{2}$-treated cells were also significantly suppressed by LYR-8. However, LYR8 alone in the absence of $\mathrm{CoCl}_{2}$ did not significantly alter the basal expression of VEGF and COX-2 or $\mathrm{PGE}_{2}$ production. When we searched for the upstream signaling molecules responsible for regulating HIF- $1 \alpha$ activation and inducing angiogenesis, we observed that LYR-8 effectively suppressed Akt signaling but not ERK signaling (Fig. 4I). As shown in Fig. 4J, LY294002, a PI3K/Akt inhibitor, and U0126, a MEK inhibitor, suppressed $\mathrm{CoCl}_{2}$-induced HIF-1 $\alpha$ accumulation in the same manner as LYR-8.

\section{DISCUSSION}

Cannabinoid-based therapies are slowly being introduced for the palliative treatment of pain in cancer patients. Many studies have also focused on cannabinoids as potential antitumor agents. $^{20-24)}$ Initially, cannabinoids were reported to regulate cancer cell growth and apoptosis ${ }^{25,26)}$ and to suppress tumor neovascularization. ${ }^{22,24)}$ However, their clinical usage is severely restricted by their psychotropic natures, thus, novel approaches are required to identify cannabinoids suitable for the treatment of cancer. We searched for potent, non-psychoactive candidate molecules and synthesized novel hexahydrocannabinol analogs that are structurally similar to tetrahydrocannabinol. In the present study, we clearly demonstrated that LYR-8 suppresses cancer-induced angiogenesis and retards tumor growth in vivo. LYR-8 was found to be well tolerated during this study as no differences in body weight between vehicle and LYR-8 treated animals or evidence of overt toxicity were observed. Furthermore, we uncovered the detailed mechanism of action of LYR-8 by showing that LYR8 inhibited the expression level of HIF- $1 \alpha$ in tumor tissues in both a xenograft mouse model and cancer cells in vitro.

It has been suggested that the interaction between cancer cells and the tumor microenvironment plays a critical role in cancer growth, invasion, metastasis, and angiogenesis. ${ }^{27-29)}$ VEGF serves as a regulatory factor for the cancer microenvironment as emphasized in many studies showing a strong correlation between increased VEGF levels, angiogenesis and tumor progression. ${ }^{30,31)}$ Moreover, bevacizumab, anti-VEGF antibody, has proven successful in clinical trials, ${ }^{32)}$ and optimal regimens have been developed for the agents in metastatic colorectal cancer. ${ }^{33)}$ However, because tumors have the ability to secrete a variety of proangiogenic factors in tumor microenvironments, they can circumvent single-target therapies and resume aggressive growth. Recent anti-angiogenic anti-tumor drug development efforts have focused on molecules that target multiple regulatory points of the tumor microenvironment. In the present study, we showed that LYR- 8 significantly suppressed VEGF expression in tumor tissues dissected from both CAMs and mouse xenografts. VEGF expression in HT-29 cells under $\mathrm{CoCl}_{2}$-induced hypoxic conditions was also significantly suppressed by LYR-8, while LYR-8 alone had little 


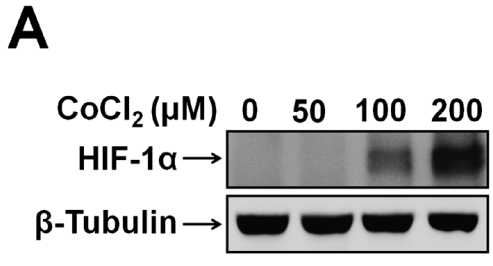

D
B

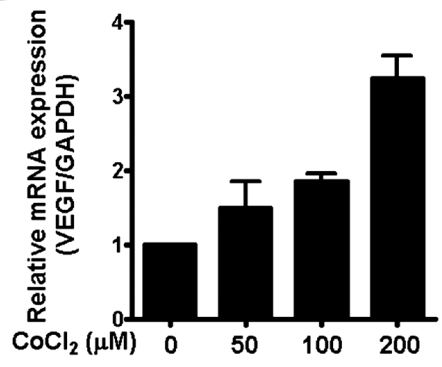

C

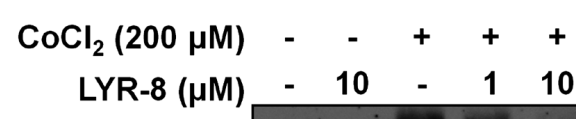

LYR-8 $(\mu \mathrm{M}) \quad-10 \quad-\quad 110$

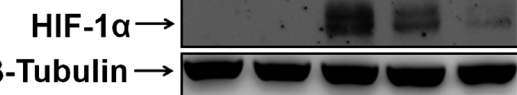

$H I F-1 \alpha \rightarrow----$

GAPDH $\longrightarrow \longrightarrow$

$\mathbf{F}$

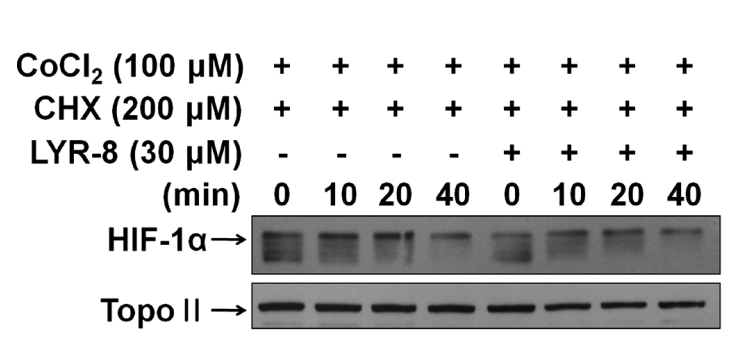

E

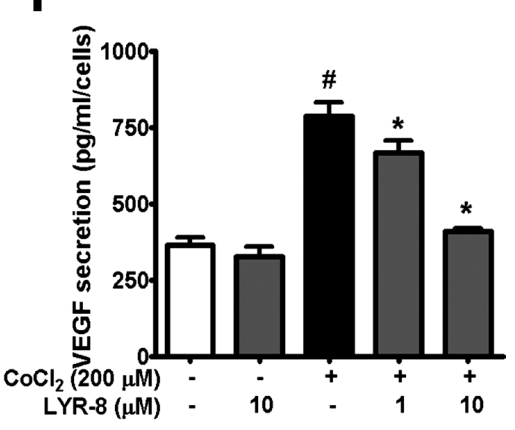

G

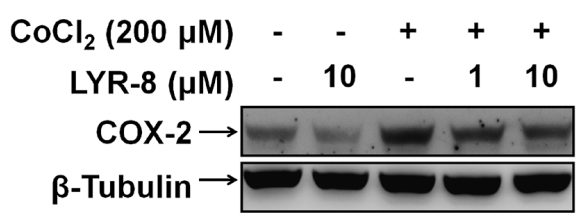

I

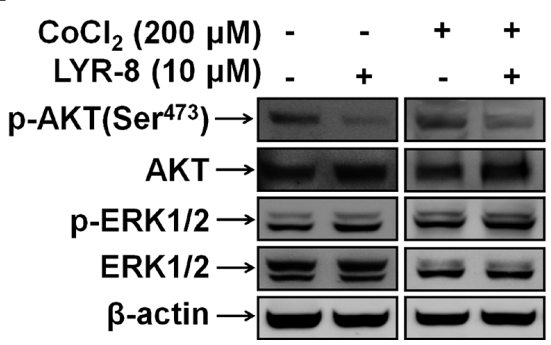

H

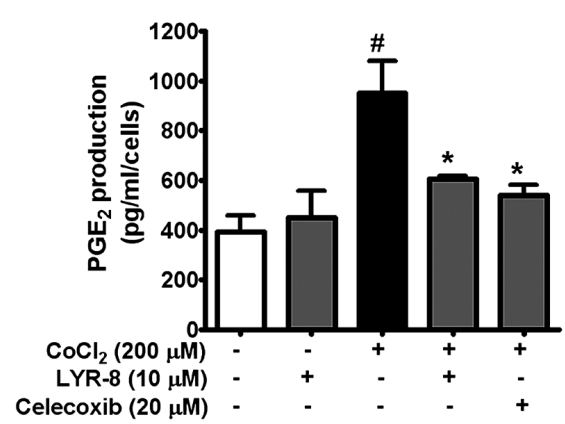

J

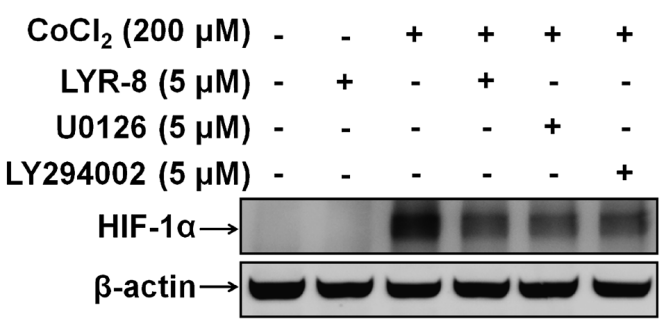

Fig. 4. Involvement of HIF-1 $\alpha$ in LYR-8-Mediated Downregulation of VEGF and COX-2

Hypoxia mimetic $\mathrm{CoCl}_{2}$-activated HT-29 cells were used as an in vitro model to study LYR-8-mediated downregulation of VEGF and COX-2. (A) Western blot analysis showing a concentration-dependent increase in HIF- $1 \alpha$ levels in HT-29 cells exposed to $\mathrm{CoCl}_{2}$ (16h). (B) Real-time PCR analysis showing VEGF mRNA expression in HT29 cells exposed to $\mathrm{CoCl}_{2}$ (16h). (C) After HT-29 cells were treated with $\mathrm{CoCl}_{2}$ for $16 \mathrm{~h}$ in the presence or absence of LYR-8, HIF-1 $\alpha$ protein and mRNA level were analyzed (C). To examine HIF-1 $\alpha$ stability change, HT-29 cells were either untreated or pretreated with $\mathrm{CoCl}_{2}(100 \mu \mathrm{M})$ for $4 \mathrm{~h}$ followed by addition of cycloheximide $(\mathrm{CHX}$; $200 \mu \mathrm{M})$ for the indicated times. HIF-1 $\alpha$ level in nuclear extracts were visualized by Western blot analysis (D). (E-J) Cells were treated the same as described in (C), and then, analyzed for VEGF mRNA by qRT-PCR (E), VEGF protein secretion (F) and PGE $_{2}$ production (H) by ELISA, COX-2 protein (G) and the activation of ERK and Akt by Western blot (I). The effects of U0126 (a MEK inhibitor) or LY294002 (a PI3-kinase inhibitor) on HIF-1 $\alpha$ expression were also assessed by Western blot (J). ${ }^{*}<0.05$, compared to the vehicle-treated control group, ${ }^{*} p<0.05$, compared to the $\mathrm{CoCl}_{2}$-treated group. These experiments are representative of 3 independent experiments.

effect on basal VEGF expression in HT-29 cells.

In addition to its effect on VEGF, LYR-8 also inhibits COX-2. Recently, Cox-2 has been identified as a direct target for HIF-1 $\alpha$, and its up-regulation was found to contribute to tumor survival and angiogenesis. ${ }^{34,35)} \mathrm{COX}-2$ is expressed in a wide range of cells including cancer cells, endothelial cells, immune cells, and stromal fibroblasts within tumors. In addition, cancer-associated stromal cells stimulate COX-2 expression in cancers by releasing soluble factors. ${ }^{36)} \mathrm{PGs}$, the products of COX-2 enzyme activity in cancer cells, may induce the transformation of stromal cells to optimized forms better suited to the cancer microenvironment. ${ }^{15,37)}$ Accordingly, 
COX-2 is also considered a promising therapeutic target for cancer treatment and prevention. In the present study, it was found that the high level of COX-2 in hypoxic tumor tissues in vivo was dramatically reduced by LYR-8. These results suggest that LYR-8 effectively interferes with the cancermicroenvironment interaction. In addition, the suppression of PGE $_{2}$ production by LYR-8 also demonstrated in HT-29 cells treated with $\mathrm{CoCl}_{2}$, which further supports the notion that the inhibitory action of LYR-8 on tumor growth and angiogenesis is mediated through regulation of the tumor microenvironment such as COX-2 and $\mathrm{PGE}_{2}$ production. It is necessary to mention the differential inhibitory effects of LYR- 8 on VEGF and COX-2 expression between our in vivo studies (Fig. 3C) and in vitro cell culture (Figs. 4C,D). The cell culture condition does not truly represent in vivo tumor study, where tumor tissues also contain endothelial cells and pericytes. Previously, we reported that LYR-8 causes dose-dependent inhibition of cancer cell growth, ${ }^{15)}$ which is cell density dependent. ${ }^{16)}$ In the present study, we did a careful normalization of our all immunoassays utilizing conditioned media with cell viability to rule out the effects of toxicity. Unexpectedly, the seeming reduction in the level of VEGF and PGE $_{2}$ production in LYR-8-treated group turned out to be insignificant after careful normalization and further repetition in independent experiments using immunoassays and qRT-PCR. Thus, we firmly conclude that LYR-8 did not alter basal expression of VEGF and COX-2, or $\mathrm{PGE}_{2}$ production. In $\mathrm{CoCl}_{2}$-treated $\mathrm{HT}-29$ cells to mimic, at least in some extent, the hypoxic condition as in tumors, LYR-8 suppressed $\mathrm{CoCl}_{2}$-induced HIF-1 $\alpha$, and subsequently its targets, VEGF and COX-2.

The expression of VEGF is mainly regulated by HIF- $1 \alpha$, and COX-2 is also a target of HIF- $1 \alpha$. In animal models, HIF-1 $\alpha$ overexpression is associated with increased tumor growth, vascularization, and metastasis, whereas HIF- $1 \alpha$ loss-of-function mutation has the opposite effect. ${ }^{38)}$ Therefore, various HIF-1 inhibitors have been evaluated with respect to their effects on pathological angiogenesis and cancer. Of the many HIF-1 inhibitors identified, YC-1 [3-(5-hydroxymethyl2-furyl)-1-benzylindazole ${ }^{17}$ ], PX-12 [1-methylpropyl 2-imidazolyl disulfide ${ }^{39)}$ ], P2630 [pyridylpyrimidine ${ }^{40)}$ ], and other emerging compounds such as heteroaryls and thiazolidinone compounds [for review see ref. 41], have shown promising in vitro and in vivo antitumor activity. In the present study, we showed that the reduced expression of HIF-1 $\alpha$ in tumor tissue from LYR-8-treated mice corresponded to the suppression of tumor growth and angiogenesis. Furthermore, in HT-29 cancer cells under hypoxia mimicking conditions using $\mathrm{CoCl}_{2}$ treatment, LYR-8 was found to dose-dependently inhibit HIF-1 $\alpha$ and consequently the expressions of target genes COX-2 and VEGF. These results indicate that HIF- $1 \alpha$ is the target of LYR8 action. To the best of our knowledge, this report is the first to show the HIF-1 $\alpha$ inhibitory potential of a cannabinoid-like hexahydrocannabinol analog.

The mechanism by which $\mathrm{CoCl}_{2}$ induces HIF- $1 \alpha$ accumulation is mediated through inhibition of prolyl hydroxylase and ubiquitin/proteosomal pathway, which stabilizes HIF-1 $\alpha$ protein. Since LYR-8 did not affect HIF- $1 \alpha$ mRNA expression (Fig. 4C) and protein stability (Fig. 4D) in $\mathrm{CoCl}_{2}$-treated cells, the significant suppression of $\mathrm{CoCl}_{2}$-induced $\mathrm{HIF}-1 \alpha$ protein by LYR-8 indicate that LYR-8 may inhibit the protein synthesis of HIF-1 $\alpha$. These results are also related to the finding that the inhibitory activity of LYR-8 on HIF-1 $\alpha$ was associated with suppression of phosphatidylinositol 3-kinase (PI3K)/Akt signaling. Recent reports have suggested that PI3K/Akt and mitogen-activated protein kinase (MAPK)/extracellular signalregulated kinase (ERK) signaling play an important role in regulating hypoxia-induced HIF-1 $\alpha$ level by increasing HIF- $1 \alpha$ protein synthesis and consequently the expression of its target genes. $^{42-45)}$ Furthermore, it is known that COX-derived PGs enhance endothelial cell survival via Akt signaling. ${ }^{46)}$ Similar to what we observed previously, ${ }^{15)}$ we found that LYR-8 inhibits the expression of molecules associated with cell survival and proliferation such as Akt and COX-2. It is possible to conclude that the LYR-8-mediated inhibition of Akt is responsible for the inhibition of HIF- $1 \alpha$ upregulation and of VEGF and COX-2 production during hypoxia. It has been suggested that anticancer agents that simultaneously inhibit multiple tumorigenic events, such as mitogenic and cell survival signaling pathways and tumor angiogenesis, are likely to be more effective at suppressing tumor growth and progression. ${ }^{47,48)}$ In agreement with this, we suggest that LYR-8 may be a potent anti-cancer agent that targets both angiogenic and survival signaling molecules, such as VEGF and COX-2.

Taken together, our findings indicate that the anti-tumor effect of the hexahydrocannabinol analog LYR-8 is due to two distinct mechanisms, indirect inhibition of tumor angiogenesis via HIF- $1 \alpha$ and VEGF and direct inhibition of tumor cell survival via Akt and COX-2. Given the opinion that HIF1 is an attractive target for cancer therapy ${ }^{12,17,49,50)}$ and that cannabinoid-based therapies are viewed with some excitement by those in cancer research, the present study provides a new perspective regarding the potential of cannabinoid-like nonpsychoactive compounds targeting Akt and HIF-1 $\alpha$ for cancer therapy and chemoprevention.

Acknowledgments This research was supported by the Basic Science Research Program (2011-0010158) through the National Research Foundation of Korea (NRF) funded by the Ministry of Education, Science and Technology (MEST), and the Regional Technology Innovation program (RTI04-01-04) through the Korea Ministry of Knowledge and Economy.

\section{REFERENCES}

1) Carmeliet P. Angiogenesis in health and disease. Nat. Med., 9, 653-660 (2003).

2) Folkman J. Angiogenesis: an organizing principle for drug discovery? Nat. Rev. Drug Discov., 6, 273-286 (2007).

3) Ferrara N. Role of vascular endothelial growth factor in physiologic and pathologic angiogenesis: therapeutic implications. Semin. Oncol., 29 (Suppl. 16), 10-14 (2002).

4) Wang GL, Jiang BH, Rue EA, Semenza GL. Hypoxia-inducible factor 1 is a basic-helix-loop-helix-PAS heterodimer regulated by cellular O2 tension. Proc. Natl. Acad. Sci. U.S.A., 92, 5510-5514 (1995).

5) Jiang BH, Rue E, Wang GL, Roe R, Semenza GL. Dimerization, DNA binding, and transactivation properties of hypoxia-inducible factor 1. J. Biol. Chem., 271, 17771-17778 (1996).

6) Huang LE, Gu J, Schau M, Bunn HF. Regulation of hypoxiainducible factor lalpha is mediated by an $\mathrm{O}_{2}$-dependent degradation domain via the ubiquitin-proteasome pathway. Proc. Natl. Acad. Sci. U.S.A., 95, 7987-7992 (1998).

7) Birner P, Schindl M, Obermair A, Breitenecker G, Oberhuber G. Expression of hypoxia-inducible factor lalpha in epithelial ovarian 
tumors: its impact on prognosis and on response to chemotherapy. Clin. Cancer Res., 7, 1661-1668 (2001).

8) Zagzag D, Zhong H, Scalzitti JM, Laughner E, Simons JW, Semenza GL. Expression of hypoxia-inducible factor 1alpha in brain tumors: association with angiogenesis, invasion, and progression. Cancer, 88, 2606-2618 (2000).

9) Schindl M, Schoppmann SF, Samonigg H, Hausmaninger H, Kwasny W, Gnant M, Jakesz R, Kubista E, Birner P, Oberhuber G, Austrian Breast and Colorectal Cancer Study Group. Overexpression of hypoxia-inducible factor lalpha is associated with an unfavorable prognosis in lymph node-positive breast cancer. Clin. Cancer Res., 8, 1831-1837 (2002).

10) Harris AL. Hypoxia-A key regulatory factor in tumour growth. Nat. Rev. Cancer, 2, 38-47 (2002).

11) Brown JM. Exploiting the hypoxic cancer cell: mechanisms and therapeutic strategies. Mol. Med. Today, 6, 157-162 (2000).

12) Semenza GL. Targeting HIF-1 for cancer therapy. Nat. Rev. Cancer, 3, 721-732 (2003).

13) Pouysségur J, Dayan F, Mazure NM. Hypoxia signalling in cancer and approaches to enforce tumour regression. Nature, 441, 437-443 (2006).

14) Lee YR, Xia LK. Efficient one-pot synthetic approaches for cannabinoid analogues and their application to biologically interesting $(-)$-hexahydrocannabinol and (+)-hexahydrocannabinol. Tetrahedron Lett., 49, 3283-3287 (2008).

15) Thapa D, Babu D, Park MA, Kwak MK, Lee YR, Kim JM, Kwon TK, Kim JA. Induction of p53-independent apoptosis by a novel synthetic hexahydrocannabinol analog is mediated via Sp1-dependent NSAID-activated gene-1 in colon cancer cells. Biochem. Pharmacol., 80, 62-71 (2010).

16) Thapa D, Lee JS, Heo SW, Lee YR, Kang KW, Kwak MK, Choi HG, Kim JA. Novel hexahydrocannabinol analogs as potential anticancer agents inhibit cell proliferation and tumor angiogenesis. Eur. J. Pharmacol., 650, 64-71 (2011).

17) Yeo EJ, Chun YS, Cho YS, Kim J, Lee JC, Kim MS, Park JW. YC1: a potential anticancer drug targeting hypoxia-inducible factor 1 . J. Natl. Cancer Inst., 95, 516-525 (2003).

18) Park BC, Park SY, Lee JS, Mousa SA, Kim JT, Kwak MK, Kang KW, Lee ES, Choi HG, Yong CS, Kim JA. The anti-angiogenic effects of 1-furan-2-yl-3-pyridin-2-yl-propenone are mediated through the suppression of both VEGF production and VEGF-induced signaling. Vascul. Pharmacol., 50, 123-131 (2009).

19) Kim TH, Hur EG, Kang SJ, Kim JA, Thapa D, Lee YM, Ku SK, Jung Y, Kwak MK. NRF2 blockade suppresses colon tumor angiogenesis by inhibiting hypoxia-induced activation of HIF-1 $\alpha$. Cancer Res., 71, 2260-2275 (2011).

20) Galve-Roperh I, Sánchez C, Cortés ML, Gómez del Pulgar T, Izquierdo M, Guzmán M. Anti-tumoral action of cannabinoids: involvement of sustained ceramide accumulation and extracellular signal-regulated kinase activation. Nat. Med., 6, 313-319 (2000).

21) Sánchez C, de Ceballos ML, Gomez del Pulgar T, Rueda D, Corbacho C, Velasco G, Galve-Roperh I, Huffman JW, Ramón y Cajal S, Guzmán M. Inhibition of glioma growth in vivo by selective activation of the $\mathrm{CB}(2)$ cannabinoid receptor. Cancer Res., 61, 5784-5789 (2001).

22) Freimuth N, Ramer R, Hinz B. Antitumorigenic effects of cannabinoids beyond apoptosis. J. Pharmacol. Exp. Ther., 332, 336-344 (2010).

23) Blázquez C, Salazar M, Carracedo A, Lorente M, Egia A, González-Feria L, Haro A, Velasco G, Guzmán M. Cannabinoids inhibit glioma cell invasion by down-regulating matrix metalloproteinase-2 expression. Cancer Res., 68, 1945-1952 (2008).

24) Blázquez C, Casanova ML, Planas A, Gómez Del Pulgar T, Villanueva C, Fernández-Aceñero MJ, Aragonés J, Huffman JW, Jorcano JL, Guzmán M. Inhibition of tumor angiogenesis by cannabinoids. FASEB J., 17, 529-531 (2003).
25) Guzmán M, Sánchez C, Galve-Roperh I. Cannabinoids and cell fate. Pharmacol. Ther., 95, 175-184 (2002).

26) Bifulco M, Di Marzo V. Targeting the endocannabinoid system in cancer therapy: a call for further research. Nat. Med., 8, 547-550 (2002).

27) Liao D, Luo Y, Markowitz D, Xiang R, Reisfeld RA. Cancer associated fibroblasts promote tumor growth and metastasis by modulating the tumor immune microenvironment in a 4T1 murine breast cancer model. PLoS ONE, 4, e7965 (2009).

28) Pietras K, Ostman A. Hallmarks of cancer: interactions with the tumor stroma. Exp. Cell Res., 316, 1324-1331 (2010).

29) Sharma M, Beck AH, Webster JA, Espinosa I, Montgomery K, Varma S, van de Rijn M, Jensen KC, West RB. Analysis of stromal signatures in the tumor microenvironment of ductal carcinoma in situ. Breast Cancer Res. Treat., 123, 397-404 (2010).

30) Jubb AM, Harris AL. Biomarkers to predict the clinical efficacy of bevacizumab in cancer. Lancet Oncol., 11, 1172-1183 (2010).

31) Ferrara N. VEGF and the quest for tumour angiogenesis factors. Nat. Rev. Cancer, 2, 795-803 (2002).

32) Jain RK, Duda DG, Clark JW, Loeffler JS. Lessons from phase III clinical trials on anti-VEGF therapy for cancer. Nat. Clin. Pract. Oncol., 3, 24-40 (2006).

33) de Gramont A, Larsen AK, Tournigand C, Louvet C, André T, GERCOR (French Oncology Research Group). Update on targeted agents for adjuvant treatment of colon cancer in 2006. Gastrointest Cancer Res, 1 (Suppl. 2), S47-S49 (2007).

34) Kaidi A, Qualtrough D, Williams AC, Paraskeva C. Direct transcriptional up-regulation of cyclooxygenase-2 by hypoxia-inducible factor (HIF)-1 promotes colorectal tumor cell survival and enhances HIF-1 transcriptional activity during hypoxia. Cancer Res., 66, 6683-6691 (2006).

35) Lee JJ, Natsuizaka M, Ohashi S, Wong GS, Takaoka M, Michaylira CZ, Budo D, Tobias JW, Kanai M, Shirakawa Y, Naomoto Y, Klein-Szanto AJ, Haase VH, Nakagawa H. Hypoxia activates the cyclooxygenase-2-prostaglandin E synthase axis. Carcinogenesis, 31, 427-434 (2010).

36) Tjiu JW, Chen JS, Shun CT, Lin SJ, Liao YH, Chu CY, Tsai TF, Chiu HC, Dai YS, Inoue H, Yang PC, Kuo ML, Jee SH. Tumorassociated macrophage-induced invasion and angiogenesis of human basal cell carcinoma cells by cyclooxygenase-2 induction. $J$. Invest. Dermatol., 129, 1016-1025 (2009).

37) Yashiro M, Nakazawa K, Tendo M, Kosaka K, Shinto O, Hirakawa K. Selective cyclooxygenase-2 inhibitor downregulates the paracrine epithelial-mesenchymal interactions of growth in scirrhous gastric carcinoma. Int. J. Cancer, 120, 686-693 (2007).

38) Semenza GL. Evaluation of HIF-1 inhibitors as anticancer agents. Drug Discov. Today, 12, 853-859 (2007).

39) Welsh SJ, Williams RR, Birmingham A, Newman DJ, Kirkpatrick DL, Powis G. The thioredoxin redox inhibitors 1-methylpropyl 2-imidazolyl disulfide and pleurotin inhibit hypoxia-induced factor lalpha and vascular endothelial growth factor formation. Mol. Cancer Ther., 2, 235-243 (2003).

40) Yewalkar N, Deore V, Padgaonkar A, Manohar S, Sahu B, Kumar P, Jalota-Badhwar A, Joshi KS, Sharma S, Kumar S. Development of novel inhibitors targeting HIF-1 $\alpha$ towards anticancer drug discovery. Bioorg. Med. Chem. Lett., 20, 6426-6429 (2010).

41) Ban HS, Uto Y, Nakamura H. Hypoxia-inducible factor inhibitors: a survey of recent patented compounds (2004-2010). Expert Opin. Ther. Pat., 2, 131-146 (2010).

42) Alvarez-Tejado M, Naranjo-Suarez S, Jiménez C, Carrera AC, Landázuri MO, del Peso L. Hypoxia induces the activation of the phosphatidylinositol 3-kinase/Akt cell survival pathway in PC12 cells: protective role in apoptosis. J. Biol. Chem., 276, 22368-22374 (2001).

43) Kong D, Yamori T. Phosphatidylinositol 3-kinase inhibitors: promising drug candidates for cancer therapy. Cancer Sci., 99, 
1734-1740 (2008).

44) Terzuoli E, Donnini S, Giachetti A, Iñiguez MA, Fresno M, Melillo G, Ziche M. Inhibition of hypoxia inducible factor-lalpha by dihydroxyphenylethanol, a product from olive oil, blocks microsomal prostaglandin-E synthase-1/vascular endothelial growth factor expression and reduces tumor angiogenesis. Clin. Cancer Res., 16, 4207-4216 (2010).

45) Liu L, Ning X, Han S, Zhang H, Sun L, Shi Y, Sun S, Guo C, Yin F, Qiao T, Wu K, Fan D. Hypoxia induced HIF-1 accumulation and VEGF expression in gastric epithelial mucosa cell: involvement of ERK1/2 and PI3K/Akt. Mol. Biol. (Mosk.), 42, 459-469 (2008).

46) Gately S, Li WW. Multiple roles of COX-2 in tumor angiogenesis: a target for antiangiogenic therapy. Semin. Oncol., 31 (Suppl. 7), 2-11
(2004).

47) Yi T, Yi Z, Cho SG, Luo J, Pandey MK, Aggarwal BB, Liu M. Gambogic acid inhibits angiogenesis and prostate tumor growth by suppressing vascular endothelial growth factor receptor 2 signaling. Cancer Res., 68, 1843-1850 (2008).

48) Singh RP, Gu M, Agarwal R. Silibinin inhibits colorectal cancer growth by inhibiting tumor cell proliferation and angiogenesis. Cancer Res., 68, 2043-2050 (2008).

49) Lu X, Kang Y. Hypoxia and hypoxia-inducible factors: master regulators of metastasis. Clin. Cancer Res., 16, 5928-5935 (2010).

50) Monti E, Gariboldi MB. HIF-1 as a target for cancer chemotherapy, chemosensitization and chemoprevention. Curr. Mol. Pharmacol., 4, 62-77 (2011). 